\title{
Gestação Trigemelar Espontânea: Complicações Maternas e Resultados Perinatais
}

\author{
Natural Triplet Pregnancies: Maternal Complications and Perinatal Results \\ Maria Regina Torloni, Márcia Akemi Kikuti, Márcia Maria Marques da Costa
}

\section{RESUMO}

Objetivo: avaliar as complicações maternas e os resultados perinatais de gestações trigemelares. Métodos: estudo retrospectivo analisando variáveis maternas e perinatais de todas gestações trigemelares com peso fetal $\geq 500 \mathrm{~g}$ ocorridas em 8 anos na Maternidade Escola de Vila Nova Cachoeirinha.

Resultados: entre 1990-1998, 18 gestantes deram à luz trigemelares, representando 1:2.060 partos. As principais complicações foram a prematuridade $(94,4 \%)$ e pré-eclâmpsia (44,4\%) e 83,3\% das pacientes precisaram ser internadas antes do parto por 1-50 dias, a maioria para inibição de trabalho de parto prematuro. O parto foi cesáreo em 88,9\%, a IG média ao nascer foi $34,2$ semanas $( \pm 1,8)$, o peso médio foi $1.827 \mathrm{~g}$ ( \pm 421$)$, com $20,4 \%<1.500 \mathrm{~g}$ e $75,9 \%$ pesando entre 1.500-2.499 g. Houve discordância de peso ( $\geq 25 \%$ ) em 38,9\% das gestações e 35,2\% dos 54 conceptos eram pequenos para idade gestacional. A morbidade neonatal acometeu 86\% dos nativivos e 3,7\% tiveram malformações evidentes. A mortalidade perinatal foi de 16,7\%: 7,4\% natimortos e 9,3\% neomortos. A permanência média no berçário foi 18,5 dias e a principal causa de neomortalidade foi a sepse tardia.

Conclusão: as gestações trigemelares tiveram elevada incidência de complicações obstétricas, exigiram internações maternas prolongadas e terminaram quase todas em parto operatório. A nati e neomortalidade foram elevadas, a morbidade neonatal acometeu praticamente todos os neonatos e a permanência no berçário foi prolongada, expondo esses prematuros à infecção, principal causa de mortalidade neonatal. A gestação trigemelar é de alto risco materno e fetale exige atendimento em centros terciários.

PALAVRAS-CHAVE: Trigemelaridade. Gestação múltipla. Óbito fetal. Natimortalidade.

\section{Introdução}

Até a década de 70 , a gestação tripla era um fenômeno exclusivamente espontâneo e bastante raro, ocorrendo em 1:5.302 até 1:10.415 partos $^{1}$, variando conforme fatores genéticos e geográficos. Desde então, com o advento das técnicas de reprodução assistida, ocorreu um aumento mundial na incidência das gestações múltiplas, e nas últimas duas décadas a trigemelaridade é encontrada em uma proporção de 1:2083 a 1:849 partos $^{2}$. Comparadas às gestações únicas e gemelares, as trigemelares apresentam mais complicações maternas (anemia, préeclâmpsia, parto operatório), alem de elevadas taxas de prematuridade e mortalidade perinatal,

Maternidade Escola de Vila Nova Cachoeirinha, São Paulo. SP Correspondência: Maria Regina Torloni

Rua Fagundes Dias, 172, ap. 31

04055-000 - São Paulo - SP chegando alguns autores a preconizar a redução embrionária seletiva precoce nessas pacientes, visando assegurar um melhor prognóstico para os sobreviventes ${ }^{3-5}$.

Por tratar-se de gestação de alto risco cuja freqüência vem crescendo mundialmente, o estudo da trigemelaridade é um assunto atual e relevante. A coleta de dados da nossa realidade, com gestantes de baixo nível socioeconômico e que dispõem de limitados recursos médicos, permite a obtenção de informações úteis na orientação dos casais que enfrentam esta situação, alem de possibilitar o planejamento de estratégias assistenciais mais eficazes.

\section{Pacientes e Método}

Este foi um estudo retrospectivo descritivo 
de todos os casos de gestações triplas, com peso fetal de pelo menos um dos fetos $(\geq 1)$ maior que $499 \mathrm{~g}$, que tiveram seus partos na Maternidade Escola de Vila Nova Cachoeirinha (MEVNC) entre $1 / 6 / 90$ e $30 / 6 / 98$. Situada na periferia da zona norte de São Paulo, esta instituição é uma maternidade municipal (gratuita) especializada em gestação de alto risco. Conta com programa de residência médica em gineco-obstetrícia e neonatologia e realiza 4.500-5.000 partos por ano. A clientela é constituída por pacientes de baixo nivel socioeconômico, provenientes de toda a cidade e de vários municípios vizinhos. Apesar de não existir um protocolo específico para gestações múltiplas, é norma desta instituição inibir trabalho de parto prematuro (TPP) de todas gestantes com idade gestacional (IG) inferior a 34 semanas, utilizando-se terbutalina, eventualmente associada a inibidores da sintese de prostaglandinas (indometacina, aspirina) por até 5 dias. Emprega-se também corticóide (betametasona ou dexametasona $24 \mathrm{mg} /$ semana) até a 34a semana. Uma vez inibida a dinâmica uterina, a paciente é transferida para a Casa da Gestante de Alto Risco (CGAR), enfermaria situada dentro do complexo hospitalar da Maternidade, que dispõe de 20 leitos destinados a internações obstétricas prolongadas ( $\geq 3$ dias). Na CGAR as gestantes são acompanhadas diariamente pela mesma equipe multidisciplinar (médicos, enfermeiras, assistente social, nutricionista e psicóloga), permanecendo internadas até o parto ou alta, conforme as condições individuais de cada caso.

Os prontuários do estudo foram selecionados por meio dos livros de registro de partos. As variáveis maternas analisadas foram idade, paridade, complicações obstétricas e clínicas, internação durante a gestação, tipo de parto, morbidade puerperal e mortalidade. O TPP foi definido como a presença de contrações regulares antes da $37 \underline{\text { a }}$ semana, associada ou não à modificações cervicais, que exigiu o uso de tocoliticos parenterais (EV ou SC). Anemia foi definida como detecção de níveis de hemoglobina $<11 \mathrm{mg} / \mathrm{dl}$, e a rotura prematura de membranas (RPM) como a perda de líquido amniótico doze ou mais horas antes do início das contrações. As variáveis perinatais foram a IG, peso, retardo de crescimento (RCIU), discordância fetal (peso maior - peso menor/peso maior $\geq 25 \%$ ), Apgar de 1 은 e 5o minutos, morbidade neonatal, anomalias congênitas, tempo de permanência no berçário e mortalidade perinatal. A IG foi baseada na data da última menstruação e/ou em ultra-sonografia de 1 e e $2^{\circ}$ trimestre e foi sempre confirmada no berçário pelo exame no recém-nascido (RN) usando o método de Capurro. Nos casos com discrepância maior que 2 semanas entre a IG obstétrica e pediátrica, foi utilizado o valor da última. Os neonatos com peso situado abaixo do percentil 10 para sua IG (curva própria da Maternidade) foram classificados como pequenos para a idade gestacional (PIG).

Para facilitar o manuseio dos dados, as variáveis coletadas foram colocadas no programa Epi Info 6 versão 3.1 .

\section{Resultados}

No período do estudo ocorreram 37.084 partos e foram identificadas 18 gestações triplas, todas espontâneas, que preencheram os critérios de inclusão, o que representa uma incidência de 1:2.060 partos.

A Tabela 1 apresenta as principais características maternas. A idade materna variou de 21 a 39 anos (média $28,1 \pm 5,0$ ), com $66,6 \%$ das pacientes concentradas entre 25-34 anos e $27,8 \%$ com idade inferior. A paridade variou de 0 até 7, com $33,3 \%$ de nulíparas $(6 / 18)$ e $11,1 \%$ das pacientes com $\geq 3$ partos. Todas as pacientes tiveram ao menos uma das complicações obstétricas ou clínicas mencionadas na Tabela 1 .

Tabela 1 - Aspectos maternos em 18 casos com gestações trigemelares espontâneas.

\begin{tabular}{lrr}
\hline & $\mathbf{n}$ & $\%$ \\
\hline Complicações obstétricas * & 11 & 61,1 \\
TPP & 8 & 44,4 \\
Pré-eclâmpsia & 6 & 33,3 \\
RPM & & \\
Complicações clínicas * & 8 & 44,4 \\
Anemia & 5 & 27,8 \\
ITU & 2 & 11,1 \\
HAC & 15 & 83,3 \\
Internação na gestação & & \\
Idade gestacional no parto & & 44,4 \\
$31-33$ semanas & 8 & 50,0 \\
$34-36$ semanas & 9 & 5,6 \\
>37 semanas & 1 &
\end{tabular}

*Várias pacientes apresentaram mais de uma complicação.

TPP: trabalho de parto prematuro. RPM: rotura prematura de membranas.

ITU: infecção do trato urinário. HAC: hipertensão arterial crônica.

A gestação tripla foi corretamente diagnosticada pela ultra-sonografia em 14 pacientes $(78 \%)$, ao passo que 4 tinham laudos de gestação dupla, até o momento do parto.

A prematuridade foi a principal intercorrên- 
cia: apenas 1 paciente alcançou a 37a semana, (após internação de 47 dias, motivado pelo risco de rotura uterina devido a 2 cesáreas anteriores). A IG variou de 31-37 semanas (média de $34,2$ semanas $\pm 1,8)$, com apenas um parto antes da 32a semana. Em 6 casos $(33,3 \%)$ a prematuridade foi eletiva: em 3 gestantes devido à préeclâmpsia grave (32-34 semanas), em 2 após a constatação de morte fetal (33-34 semanas) e uma em decorrência do risco de rotura uterina (antecedente de 4 cesáreas, sendo a resolução indicada após amniocentese na 34aㅗ semana evidenciando maturidade fetal).

A prematuridade foi espontânea em 11 pacientes $(61,1 \%)$, das quais 3 chegaram à MEVNC em trabalho de parto avançado, uma em expulsivo, com IG de 31,31 e 34 semanas, respectivamente. Desses 9 RN, 2 morreram $(22,2 \%)$. Cinco gestantes foram atendidas em TPP inicial com 28, 30, 31, 31 e 32 semanas, receberam tocoliticos e corticóides, sem efeitos colaterais significantes, e permaneceram internadas em repouso na CGAR até o parto. A terapêutica prolongou estas gestações em 33, 3, 24, 4 e 29 dias, respectivamente. Dos $15 \mathrm{RN}, 13$ sobreviveram (mortalidade de 13,3\%). Os dois neomortos eram irmãos (casos 1 e 2 da Tabela 3). Três outras pacientes foram internadas preventivamente na CGAR com 27, 30 e 30 semanas devido a alterações cervicais silenciosas (sem contrações). Receberam corticóides e permaneceram em repouso por 41, 44 e 21 dias, quando ocorreu rotura prematura das membranas, entrando subseqüentemente em TPP, não sendo inibidas. Esses 9 RN sobreviveram.

Ao todo 15 pacientes (83,3\%) ficaram internadas entre 1-50 dias antes do parto (média $21,9$ dias $\pm 18,2)$, sendo um terço por até 7 dias e um terço por $\geq 30$ dias. Os motivos foram TPP inibido (5 casos), pré-eclâmpsia grave (4), cérvicodilatação silenciosa (3) e risco de rotura uterina devido ao antecedente de cesáreas (3).

A cesariana foi realizada em 16 dos 18 casos $(88,9 \%)$ sendo as indicações: gestação múltipla na presença de trabalho de parto (5), préeclâmpsia (4), iteratividade (4) e TP com apresentação anômala do primeiro feto (3). Nenhuma paciente apresentou hemorragia significante ou atonia uterina periparto. Duas puérperas tiveram infecção (1 endometrite e 1 de parede) e não ocorreu nenhum óbito materno.

A placentação foi tricoriônica em 5 casos $(27,8 \%)$ e esses 15 fetos nasceram vivos. Em 8 casos $(44,4 \%)$ existiam 2 placentas. Neste grupo, os 8 fetos que possuiam sua própria placenta foram nativivos e 2 dos 16 fetos que compartilhavam placentas morreram intra-útero. Em 5 casos $(27,8 \%)$ a placenta era única e 2 desses 15 fetos nasceram mortos. A taxa de natimortalidade portanto foi nula para fetos com sua própria placenta, e de $12,9 \%$ (4/31) para os fetos que compartilhavam a sua placenta com um ou dois fetos. A Tabela 2 apresenta os resultados perinatais dos $54 \mathrm{RN}$. O peso médio foi de 1.827 g $( \pm 421)$, variando de $1.020-2.640 \mathrm{~g}$. O peso ao nascer do menor sobrevivente foi de $1.070 \mathrm{~g} \mathrm{e}$ todos os nativivos com mais de $1.350 \mathrm{~g}$ sobreviveram. Mais de um terço das crianças (19/54) mostravam evidência de RCIU, sendo classificadas como PIG. A diferença entre os pesos fetais foi superior a $25 \%$ em 7 das 18 gestações $(38,9 \%)$.

Tabela 2 - Características perinatais de 54 trigemelares.

\begin{tabular}{|c|c|c|}
\hline & $\mathrm{n}$ & $\%$ \\
\hline \multicolumn{3}{|l|}{ Peso ao nascer } \\
\hline$<1.500 \mathrm{~g}$ & 11 & 20,4 \\
\hline $1.500-2.499 \mathrm{~g}$ & 41 & 75,9 \\
\hline$>2.499 \mathrm{~g}$ & 2 & 3,7 \\
\hline Morbidade neonatala & 43 & $86,0^{\mathrm{b}}$ \\
\hline respiratória & 36 & 72,0 \\
\hline icterícia & 30 & 60,0 \\
\hline metabólica & 20 & 40,0 \\
\hline infecciosa & 14 & 28,0 \\
\hline Mortalidade perinatal & 9 & 16,7 \\
\hline natimortalidade & 4 & 7,4 \\
\hline neomortalidade & 5 & 9,3 \\
\hline Mortalidade corrigida ${ }^{c}$ & 8 & 14,8 \\
\hline
\end{tabular}

No primeiro minuto de vida, $42 \%(21 / 50)$ dos nativivos apresentaram hipoxia (Apgar $<8$ ), sendo 20\% leve (Apgar 7), 16\% moderada (Apgar 4-6) e 6\% grave (Apgar 0-3). No 5o minuto, 6\% desses RN (3/50) ainda apresentavam hipóxia: $4 \%$ leve e $2 \%$ moderada. Dos 36 RN que apresentaram problemas respiratórios, 3 tiveram membrana hialina e 33 apresentaram desconforto respiratório adaptativo. A hipoglicemia foi o distúrbio metabólico mais freqüente (10 casos), seguido de hipocalcemia (8 casos), e várias crianças tiveram mais de 1 intercorrência. As complicações infecciosas foram graves, evoluindo com sepse neonatal em 8 casos, com 4 óbitos (Tabela 3). Dois nativivos apresentaram malformações evidentes. O primeiro apresentava defeito ósseo nos polegares, obtendo alta sem outras intercorrências. O segundo tinha uma cardiopatia congênita complexa e atresia de esôfago; desenvolveu edema agudo pulmonar e 
morreu no $13^{\circ}$ dia de vida.

Tabela 3 - Características dos neomortos em 54 trigemelares.

\begin{tabular}{|c|c|c|c|c|c|c|}
\hline Caso & Peso & IG (semanas) & PIG & Discordância (peso fetal) & Época (dias pós-parto) & Causa \\
\hline $1^{*}$ & 1.020 & 31 & $\operatorname{sim}$ & $\operatorname{sim}$ & 37 & sepse \\
\hline $2^{*}$ & 1.030 & 31 & $\operatorname{sim}$ & $\operatorname{sim}$ & 13 & EAP, MF \\
\hline 4 & 1.220 & 34 & $\operatorname{sim}$ & $\operatorname{sim}$ & 12 & sepse \\
\hline $5^{\star \star}$ & 1.340 & 32 & não & não & 18 & sepse \\
\hline
\end{tabular}

EAP: edema agudo de pulmão. MF: malformação fetal (cardíaca e esofágica). PIG: pequeno para a idade gestacional.

${ }^{*} 2^{\circ}$ e $3 \circ$ gemelares da mesma paciente. ${ }^{* *} 10$ e $3 \circ$ gemelares da mesma paciente.

A permanência no berçário variou de 3 até 83 dias (média de 18,5 dias $\pm 15,3$ ), com $28 \%$ dos 50 nativivos permanecendo 1 semana e $38 \%$ por mais de 3 semanas.

Duas gestantes tiveram 2 natimortos cada, totalizando 4 perdas fetais entre 54 conceptos $(7,4 \%)$. A primeira era uma paciente de 30 anos, G4 P2 (2 cesarianas) internada na 33 a semana por TPP, pré-eclâmpsia leve e miomatose uterina; recebeu uterolíticos e corticóide sem intercorrências. Durante a $2^{\text {a }}$ semana de internação, em cardiotocografia de rotina um feto apresentou desacelerações importantes e foi constatado o óbito dos outros dois. Submetida à cesariana de urgência, a paciente deu à luz um menino de $2.400 \mathrm{~g}$, banhado em mecônio, com Apgar 2 e 7, que possuía placenta e bolsa próprias. O RN evoluiu com desconforto respiratório adaptativo, hipoglicemia e hipomagnesemia e obteve alta em boas condições no $11^{\circ}$ dia de vida. Os natimortos, ambos do sexo feminino, pesaram $1.160 \mathrm{~g} \mathrm{e}$ $2.065 \mathrm{~g}$ e compartilhavam a mesma placenta e bolsa amniótica. Não tinham malformações evidentes, porém a família não autorizou a necropsia. A discordância entre os pesos dos natimortos $(43,8 \%)$ e monocorionicidade sugere transfusão feto-fetal como provável causa destes óbitos.

O segundo caso de morte fetal ocorreu em paciente de 32 anos, G2 P1 (1 cesariana), asmática de difícil controle, internada por TPP, broncoespasmo e oligoâmnio na $33^{a}$ semana, quando recebeu tocolíticos e corticóide. O duplo óbito foi constatado no 7을 dia da internação, durante evolução rotineira diária, sendo realizada cesariana imediatamente a seguir. O nativivo pesou $2.495 \mathrm{~g}$, teve Apgar 9 e 10, evoluindo sem intercorrências até sua alta no 7 o dia de vida. Os natimortos pesaram 2.130 e 2.515 g e não apresentavam malformações evidentes; a família não autorizou a necropsia. Os 3 fetos, todos adequados para a IG, eram masculinos, tinham 3 bol- sas e compartilhavam a mesma placenta, que pesou $1.225 \mathrm{~g}$. Não se identificou uma causa específica para estes óbitos.

Ocorreram 5 mortes pós-natais, quatro entre 12-18 dias e uma com 37 dias. Um dos óbitos foi atribuído a malformações e todos os demais RN morreram em decorrência de infecção (Tabela 3).

\section{Discussão}

Ao contrário dos trabalhos atuais em que de $68^{6}$ até $100 \%{ }^{7}$ dos casos de trigemelares decorrem de fertilização assistida, todos os casos deste estudo foram espontâneos. A incidência observada (1:2.060) foi superior à esperada na natureza, provavelmente em decorrência do caráter terciário da MEVNC.

Apenas um terço das nossas pacientes eram nuliparas, ao passo que nos trabalhos mais recentes elas representam $52,2 \%^{6}$ até $69,7 \%{ }^{7}$ dos casos, provavelmente refletindo o grande contingente de pacientes estéreis que concebem trigemelares após tratamentos especializados. A média elevada de idade $(28,1)$ foi compativel com a descrita ${ }^{2,7}$.

A complicação mais freqüente foi o TPP $(61,1 \%)$, e também o principal motivo das internações. Incluindo as antecipações eletivas, totalizamos $94,4 \%$ de prematuros, compativel com cifras entre $63,3 \%{ }^{8}$ e $97 \%{ }^{9}$ relatadas por diversos autores. A hiperdistensão uterina é apontada nas gestações múltiplas como o principal fator desencadeante das contrações prematuras. Porém, em muitas de nossas pacientes acreditamos que a anemia e as infecções urinárias provavelmente atuaram como cofatores para o TPP. Um pré-natal cuidadoso (com suplementação adequada de minerais e uroculturas periódicas) poderia colaborar para a eliminação destas pa- 
tologias e talvez reduzir a prematuridade.

Diversas condutas já foram propostas com o intuito de prolongar as gestações triplas, geralmente com casuística pequena e sem grupo controle, o que dificulta a avaliação de sua real eficácia. Alguns preconizam o repouso domiciliar desde o diagnóstico ${ }^{10}$, outros a partir da $20^{2} 2,11$, ou da $27^{\text {a }}$ semana ${ }^{12,13}$. Para Holcberg et al. ${ }^{9}$ o repouso hospitalar prolongaria a gravidez, melhoraria o fluxo uterino, aliviaria desconfortos maternos (lombalgia, dispnéia, edema) e permitiria a intervenção imediata em caso de TPP. Outros ${ }^{14}$ apontam que essas medidas não produzem melhora significante nos resultados perinatais. Apesar de nossa pequena casuistica, pudemos observar que todas as pacientes (6) internadas eletivamente (sem contrações) ultrapassaram a 33 a semana e que a única paciente que atingiu a 37 a semana pertencia a este grupo. Por outro lado, 3 das 8 pacientes com contrações no momento da internação tiveram RN com IG $<33$ semanas. Acreditamos que, para nossas gestantes de baixo nivel socioeconômico, a hospitalização é muitas vezes a única forma de garantir o repouso e a dieta balanceada que necessitam, além de facilitar o tratamento correto da anemia e o controle pressórico das hipertensas. Mas o principal benefício da internação eletiva em nosso meio talvez seja a possibilidade de agir prontamente, caso ocorram contrações, aumentando assim as chances de sucesso da tocólise. Este aspecto é muito importante em nossa realidade na qual, infelizmente, é comum atendermos gestantes em TPP com dilatação avançada, que chegam à nossa maternidade após várias horas de peregrinação pela cidade em busca de uma vaga hospitalar.

Quando o TPP estiver em fase inicial, a tocólise imediata geralmente é eficaz e prolonga a gestação por mais algum tempo, o que possibilita a aceleração da maturidade pulmonar fetal, aumentando as chances de sobrevida neonatal. Apesar deste conhecimento, na prática diária observamos que alguns obstetras, preocupados com a acentuada hipervolemia própria das gestações múltiplas, relutam em utilizar betamiméticos para a tocólise, optando por esquemas de inibição menos eficazes. Em nossos 5 casos de inibição, o uso de terbutalina EV, associada ao corticóide, não provocou reações cardiovasculares significantes. Ron-El et al. ${ }^{6}$ administraram ritodrina a 19 pacientes com gestações trigemelares, sem intercorrências. Boulot et al. ${ }^{11}$ usaram salbutamol em 33 gestações trigemelares e tiveram apenas uma complicação, em paciente submetida a cirurgia para tratamento de obstrução intestinal com 15 semanas, que rece- beu a droga profilaticamente no pós-operatório imediato e desenvolveu edema pulmonar agudo.

A elevada incidência de DHEG $(44,4 \%)$ foi semelhante à apontada pela literatura $(8 \%$ $52 \%)^{6,7,9,10,15,16}$ e está provavelmente relacionada à grande massa placentária. A DHEG é duplamente nociva, pois além de provocar danos maternos e fetais, freqüentemente leva à antecipação eletiva do parto. Em nossa casuística, a DHEG foi a responsável direta pela prematuridade em 3 dos 17 casos.

A melhor via de parto para as gestações multifetais ainda é tema controverso ${ }^{17,18}$. Nos trabalhos mais antigos o parto vaginal ocorria em até $84,7 \%^{16}$, porém o risco de morte neonatal estava diretamente relacionado à ordem de nascimento, sobretudo para os fetos em apresentação pélvica. Atualmente existe uma tendência mundial em favor da cesárea nas gestações trigemelares, justamente porque permitiria reduzir o intervalo entre os nascimentos e conseqüentemente as complicações para o segundo e terceiro fetos. Ao minimizar os tocotraumatismos e a hipoxia, a cesárea permitiria também reduzir a incidência de membrana hialina nesses prematuros ${ }^{19}$. Apesar de aumentar a morbidade materna, a cesárea também poderia proteger a mãe dos riscos associados à versão e extração conduzidas às cegas quando se opta pela via vaginal $^{9,20,21}$. Porém, ainda não existe evidência convincente de que esses beneficios sejam reais. Loucopoulos e Jewelewicz ${ }^{13}$ não observaram diferença significante no Apgar de 5 minutos entre as duas vias e só recomendam a cesárea para aqueles obstetras que não possuem experiência ou habilidade para realizar manobras como versão e extração. Wildschut et al. ${ }^{8}$ também não encontraram redução da mortalidade ou morbidade neonatal grave com o emprego da via alta, concluindo que o fator mais importante no resultado perinatal não seria a via de parto e sim a qualidade da assistência oferecida durante o TP e parto. Em nosso estudo, apesar do parto ser abdominal na quase totalidade dos casos (16/ 18 ), $42 \%$ dos RN tiveram asfixia no $1 \%$ minuto e $6 \%$ no 5o minuto de vida.

A IG $(34,2)$, o peso $(1.827 \mathrm{~g})$ médio e a incidência de complicações neonatais foram semelhantes aos descritos na literatura, porém a mortalidade perinatal $(16,7 \%)$ foi elevada comparada aos trabalhos mais recentes onde se registra taxa entre 0 e $6,6 \%{ }^{2}$.

A natimortalidade ocorreu em $11,1 \%$ de nossas gestantes $(2 / 18)$, ambas na $34^{\text {a }}$ semana. $\mathrm{Na}$ literatura este achado é relativamente freqüente nas gestações trigemelares, chegando a 
ser esperada em $12,3 \%$ dos casos, o que significa que 1 em cada 8 gestantes terá ao menos um óbito fetal ${ }^{1}$, geralmente no terceiro trimestre. Assim como nas gestações gemelares, podem existir anastomoses vasculares entre fetos que compartilham a mesma placenta, levando à transfusão feto-fetal com a morte de um ou ambos. Coincidentemente, os nossos 2 casos de óbito ocorreram em fetos que compartilhavam a mesma placenta. A menor taxa de natimortalidade observada na literatura mais recente pode, em parte, ser explicada pelo predominio absoluto de placentas tricoriônicas nesses trabalhos ${ }^{1}$, em que a maioria das gestações são trizigóticas, produto de reprodução assistida. Porém, mesmo nas gestações tricoriônicas ocorrem óbitos fetais sem uma causa específica evidente ${ }^{1}$, apesar de as pacientes estarem internadas e sob cuidadosa vigilância. A existência de RCIU em 24-62\% $\%^{9,10}$ dos trigemelares sugere que o déficit nutricional crônico pode ser um fator importante, porém ainda não está claro porque muitos fetos PIG sobrevivem até o parto, ao passo que outros morrem intra-útero. Talvez na nossa ânsia de prolongar a gestação com o intuito de assegurar maior sobrevida neonatal, acabemos por esgotar as reservas e os mecanismos adaptativos de alguns fetos, levando-os à morte intra-útero. $\mathrm{O}$ estudo sistemático da hemodinâmica fetal dos trigemelares através da dopplerfluxometria talvez possa nos ajudar a decidir até quando esperar com segurança.

Graças ao uso liberal de corticóides e aos avanços da neonatologia, a mortalidade neonatal em RN <1.500 g vem caindo continuamente nos últimos anos. Porém, após superar os problemas respiratórios críticos nos primeiros dias de vida, o RN de muito baixo peso tem ainda que enfrentar uma serie de desafios funcionais (neurológicos, metabólicos e digestivos), o que prolonga sua internação por várias semanas. A manipulação excessiva desses RN, aliada à imaturidade do seu sistema imunológico, resulta freqüentemente em infecções, por vezes fatais. $\mathrm{Na}$ literatura atual ${ }^{2}$ a principal responsável pelos óbitos neonatais em gestações trigemelares é a prematuridade extrema, que leva à mortalidade neonatal precoce. Em nossa casuística, a taxa de mortalidade neonatal foi elevada às custas da mortalidade tardia, decorrente sempre de infecção. Esta discrepância sugere que nossos neonatologistas devem se aprimorar na prevenção e no tratamento das infecções adquiridas no berçário. Concluímos que, apesar de a gestação trigemelar ter se tornado menos rara na atualidade, a experiência dos obstetras na condução desses casos ainda é limitada. As complicações clínicas e obstétricas são freqüentes, exigindo um pré-natal cuidadoso, internações prolongadas e atenção à vitalidade fetal. Ao redor da 34a semana, fetos que compartilham a mesma placenta têm maior risco de óbito intra-uterino. A prematuridade é o maior desafio e os esforços se concentram no prolongamento da gravidez, visando melhorar as chances de sobrevida dos neonatos. Em ambiente hospitalar, o uso de drogas adrenérgicas e de corticóides parece seguro para a mãe e pode beneficiar os fetos. Apesar do parto por via alta, muitos $\mathrm{RN}$ nascem deprimidos, o que compromete ainda mais o prognóstico perinatal desses prematuros. Em nossa realidade, a primeira causa de neomortalidade é a infecção tardia.

\section{SUMMARY}

Purpose: to analyze maternal complications and perinatal results of triplet pregnancies.

Method: retrospective study of maternal and perinatal data on all triplets weighing $\geq 500 \mathrm{~g}$ delivered in a period of 8 years at Maternidade Escola de Vila Nova Cachoeirinha. Results: between 1990-1998, 18 women gave birth to triplets, representing 1 in every 2,060 deliveries. The main complications were preterm delivery (94.4\%) and preeclampsia $(44.4 \%)$ and $83.3 \%$ of these patients needed hospitalization before delivery, for 1-50 days, most in order to inhibit preterm labor. Cesarean section was performed in $88.9 \%$, the mean gestational age at birth was 34.2 weeks $( \pm 1.8)$, mean weight $1,827 \mathrm{~g}( \pm 421), 20.4 \%$ weighed $<1,500 \mathrm{~g}$ and $75.9 \%$ weighed 1,500-2,499 $\mathrm{g}$. Birth weight discrepancy $(\geq 25 \%)$ occurred in $38.9 \%$ of these pregnancies and $35.2 \%$ of the 54 fetuses were small for gestational age. Eighty-six percent of live-born infants had neonatal morbidity and $3.7 \%$ had evident congenital anomalies. Perinatal mortality was 16.7\%, 7.4\% due to intrauterine demise and $9.3 \%$ due to neonatal death. The mean duration of hospitalization in the neonatal ward was 18.5 days; late neonatal sepsis was the main cause of death.

Conclusion: triplet pregnancies had high a incidence of obstetric complications, demanded prolonged maternal hospitalization and ended almost always in surgical delivery. Intrauterine and neonatal death rates were high, neonatal morbidity was detected in almost all live-born infants and their hospitalization was long, exposing these prematures to infection, their main cause of death. Triplet pregnancies carry high maternal and fetal risks and should be managed at tertiary facilities.

KEY WORDS: Triplets. Multiple pregnancies. Fetal death. 


\section{Referências}

1. Borlum KG. Third trimester fetal death in triplet pregnancies. Obstet Gynecol 1991; 77:6-9.

2. Albrecht JL, Tomich PG. The maternal and neonatal outcome of triplet gestations. Am J Obstet Gynecol 1996; 174:1551-6.

3. Berkowitz RL, Lynch L, Chitkara U, Wilkins IA, Mehalek KE, Alvarez E. Selective reduction of multifetal pregnancies in the first trimester. $\mathrm{N}$ Eng J Med 1988; 318:1043-7.

4. Wapner RJ, Davis GH, Johnson A, et al. Selective reduction of multifetal pregnancies. Lancet 1990; 335:90-3.

5. Lipitz S, Reichman B, Uval J. A prospective comparison of the outcome of triplet pregnancies managed expectantly or by multiple fetal reductions to twins. Am J Obstet Gynecol 1994; 170: 874-9.

6. Ron-El R, Caspi E, Schreyer P, Weinraub Z, Arieli S, Golberg MD. Triplet and quadruplet pregnancies and management. Obstet Gynecol 1981; 57:45863.

7. Lipitz S, Uval J, Achiron R, Schiff E, Lusky A, Reichman B. Outcome of twin pregnancies reduced from triplets compared with nonreduced twin gestations. Obstet Gynecol 1996; 87: 511-4.

8. Wildschut HI, van Roosmalern J, van Leeuwen E, Keirse MJ. Planned abdominal compared with planned vaginal birth in triplet pregnancies. $\mathrm{Br}$ J Obstet Gynaecol 1995; 102:292-6.

9. Holcberg G, Biale Y, Lewenthal H, Insler V. Outcome of pregnancy in 31 triplet gestations. Obstet Gynecol 1982; 59:472-6.

10.McFee JG, Lord EL, Jeffrey RL, et al. Multiple gestations of high fetal number. Obstet Gynecol 1974; 44:99-106.
11.Boulot P Hedon B, Pelliccia G, et al. Favourable outcome in 33 triplet pregnancies managed between 1985-1990. Eur J Obstet Gynecol Reprod Biol 1992; 43:123-9.

12.Lauersen NH, Buchman M, Beling CG. Successful quadruplet pregnancy following ovulation induced with human menopausal gonadotropin and human chorionic gonadotropin. Acta Obstet Gynecol Scand 1974; 53:387-91.

13.Loucopoulos A, Jewelewicz R. Management of multifetal pregnancies: 16-year experience at the Sloane Hospital for Women. Am J Obstet Gynecol 1982; 143:902-5.

14.Daw E. Triplet pregnancy. Br J Obstet Gynaecol 1978; 85:505-9.

15.Santema JG, Bourdrez P, Wallenburg HC. Maternal and perinatal complications in triplet compared with twin pregnancy. Eur J Obstet Gynecol Rep Biol 1995; 60:143-7.

16.Itzkowic D. A survey of 59 triplet pregnancies. Br J Obst Gynaecol 1979; 86:23-8.

17.Thiery M, Kermans G, Derom R. Triplet and higher order births: what is the optimal delivery route? Acta Genet Med Gemellol (Roma) 1988; 37:89-98.

18.Feingold M, Cetrulo C, Peters M, Chaudhury A, Shmoys S, Geifman O. Mode of delivery in multiple birth of higher order. Acta Genet Med Gemellol (Roma) 1988; 37:105-9.

19.Nochimson DJ, Petrie RH, Shah BL, Pampati N, Brunelle D. Comparison of conservative and dynamic management of premature rupture of membranes/premature labor syndrome. New approaches to the delivery of infants which may minimize the need for intensive care. Clin Perinatol 1980; 7:17-31.

20.Deale CJ, Cronje HS. A review of 367 triplet pregnancies. S Afr Med J 1984; 66:92-4.

21.Newman RB, Hamer C, Miller MC. Outpatient triplet management: a contemporary review. Am J Obstet Gynecol 1989; 161:547-55.

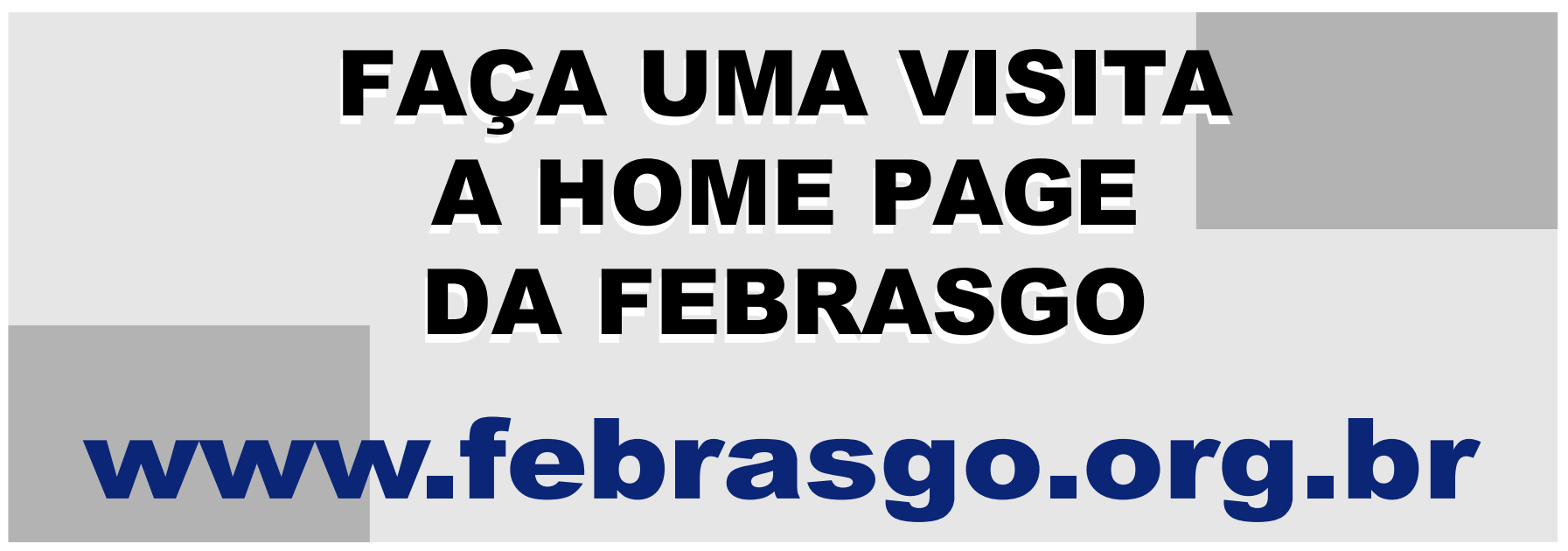

